\title{
STARS IN THE ROSAT ALL-SKY SURVEY
}

\author{
J.H.M.M. SCHMITT \\ Max-Planck-Institut für extraterrestrische Physik \\ 8046 Garching, F.R.G.
}

\section{The Stellar Content of the ROSAT All-sky Survey}

The ROSAT all-sky survey has now been completed and the analysis of this huge body of data is in progress. While final results on the number of detected $\mathrm{X}$-ray sources and their distribution in flux are not yet available, the total number of X-ray sources is expected to be around 60000 . Preliminary results from optical identifications of selected fields show that about one quarter of the $\mathrm{X}$-ray sources discovered at high galactic latitudes come from by comparison nearby stellar sources (Fleming 1991), while at lower galactic latitudes up to one half of the detected X-ray sources are of stellar origin; in areas occupied by star forming regions (for example, Orion) or open clusters (for example, Hyades or Pleiades) a large number of the detected X-ray sources can be identified with young stars, yielding up to 80 percent of the total source count as galactic stars. For the whole of the ROSAT all-sky survey we may therefore expect about one third of the total sources to be of stellar origin. The vast majority of these stellar X-ray sources is of coronal origin (i.e., late-type low mass stars). Only a relatively small number of stellar X-ray sources will be associated with early-type massive stars where the X-ray emission is thought to arise from instabilities in their radiatively driven winds or metal-poor degenerate stars where the X-ray emission comes from portions of the atmosphere considerably hotter than the optically visible photosphere. From the preliminary analyses performed so far it is already clear now that supersoft sources such as white dwarfs do not constitute a major fraction of the X-ray source population found in the ROSAT all-sky survey and the number of newly $\mathrm{X}$-ray discovered white dwarfs will certainly be considerably less than one thousand. The $\mathrm{X}$-ray emitting late-type stars are commonly referred to as "active" stars, and the ROSAT all-sky survey catalog will comprise the most extensive list of such objects.

\section{Active and Inactive Stars}

It is essential to keep in mind that X-ray selected samples of stars consist almost exclusively of such "active" stars. This can be seen through the following simple calculation: Consider the Sun as a prototype of a non-active star with a soft X-ray luminosity of $\sim 10^{27} \mathrm{erg} / \mathrm{s}$. At a flux limit of $210^{-13} \mathrm{erg} / \mathrm{cm}^{2} / \mathrm{s}$, such an object can be detected out to a distance of $9 \mathrm{pc}$; within this distance there are approximately $\sim 200$ stars. Therefore, if the Sun were indeed the paradigm for stellar X-ray emission, the stellar source content of the ROSAT all-sky survey would be almost negligible. Since this is not the case, stellar X-ray emission levels must typically be much higher than those found for the Sun. On the other hand, for the stars in the immediate solar neighborhood the ROSAT all-sky survey provides an unbiased sample of stars of all activity levels. 
This latter finding is demonstrated by a plot (cf., figure 1 ) of the ratio $f_{x} / f_{v}$ vs. $B-V$ color for "known" stars, i.e., X-ray sources identified with catalog entries in the Gliese catalog (Gliese 1959) or Bright Star Catalog (Hoffleit and Jaschek 1984). The $f_{x} / f_{v}$ ratio can be considered as an indicator of the efficiency with which X-rays can be produced in the corona of a star. As is well known from Einstein Observatory observations, the $f_{x} / f_{v}$-ratio shows significant scatter, is lowest for early-type stars and increases towards the latest stars reaching rather large values. All these results are fully confirmed by the results from the ROSAT all-sky survey.

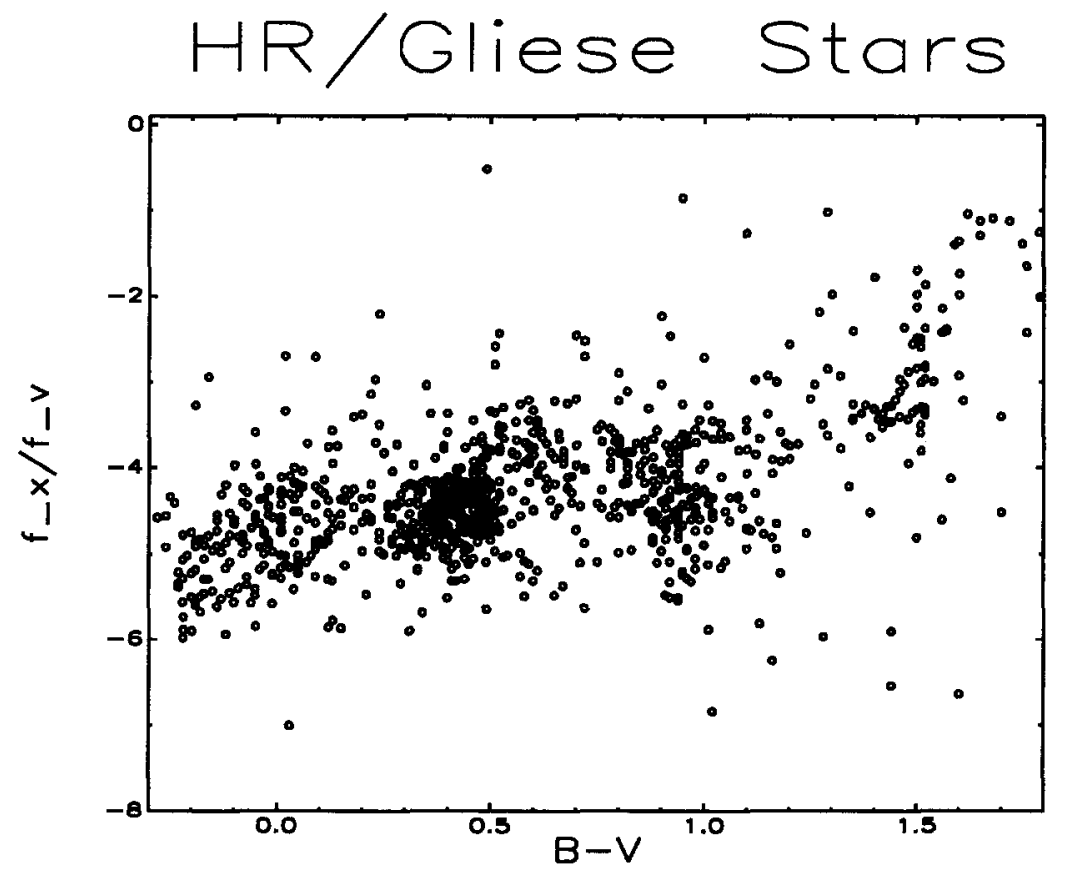

Fig.1: $f_{\mathfrak{x}} / f_{\boldsymbol{v}}$-ratio vs B-V color for a sample of bright and/or nearby stars detected in the ROSAT all-sky surevy. Note the large scatter in the $f_{x} / f_{v}$-ratio for a given spectral type.

\section{X-ray Observations of Young Galactic Clusters}

The physical basis for all the activity phenomena observed in late-type stars is usually thought to be a magnetic dynamo, which is responsible for the generation of the magnetic fields whose magnetic energy sustains the observed activity phenomena. Of considerable interest in this context is the study of the evolution of activity (as evidenced by X-ray emission) and age. Open galactic clusters are of course the targets of choice for these studies. All such clusters have been scanned during the course of the all-sky survey, allowing the detection of the more luminous X-ray sources. As an example of an open cluster I will present the all-sky survey observations of the Pleiades cluster. The Pleiades are a nearby $(\mathrm{d}=129 \mathrm{pc}$ ), young cluster (estimated age $\sim 70 \mathrm{Myrs}$, extensively studied with the Einstein Observatory, cf., Caillault and Helfand 1985, Micela et al. (1990). 
The Einstein observations had a limiting X-ray luminosity of down to $110^{29} \mathrm{erg} / \mathrm{s}$ and resulted in the detection of 85 cluster members; 69 of the X-ray detections were obtained in the core of the Pleiades where the optical information on cluster members is complete to $m_{v} \sim 17$. The all-sky survey observations of the Pleiades cluster had a sensitivity of about $2-310^{29} \mathrm{erg} / \mathrm{s}$, i.e., a factor two to three less than the Einstein observations because of the rather short exposures in the all-sky survey. Nevertheless, a total of 23 cluster members could be found as X-ray emitters in the central region of the Pleiades. In figure 2 I show a plot of $L_{X}$ vs. $B-V$ color for the detected cluster members. Interestingly, the allsky survey revealed a number of cluster members as $X$-ray sources which had not been previously detected in the Einstein observations, while on the other hand, the strongest source in the Einstein observations, i.e., Hz1136, was not detected in the ROSAT all-sky survey.

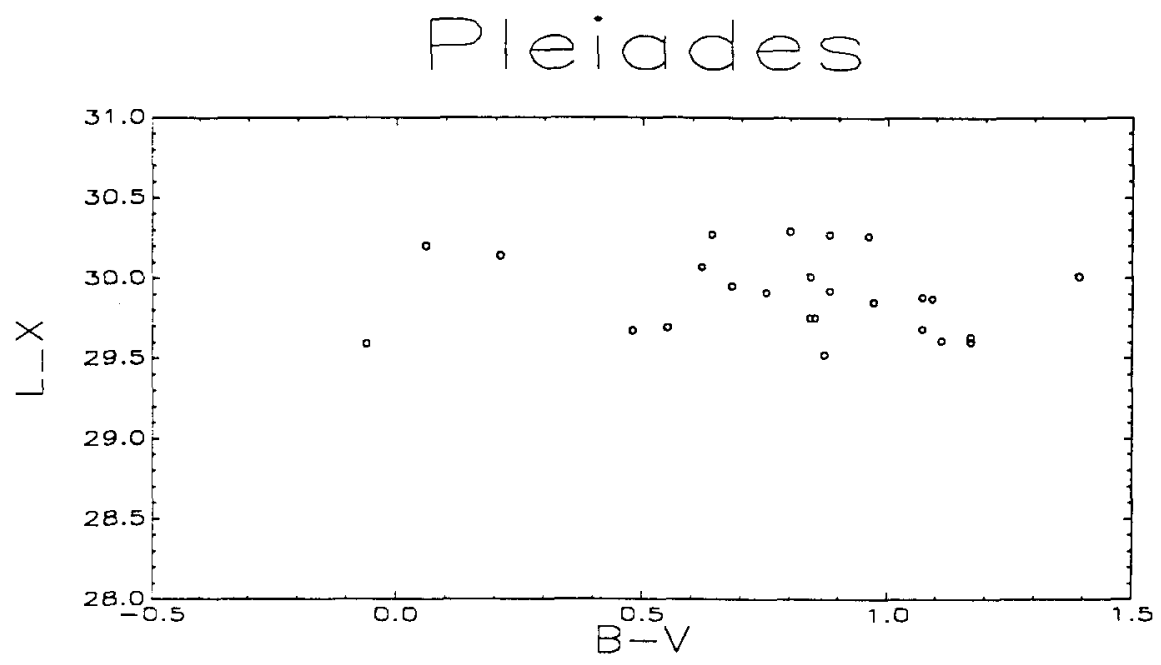

Fig.2: X-ray luminosity $L_{X}$ vs. B-V color for Pleiades members detected in the ROSA'T all-sky survey; only members in the central $2 \times 2^{\circ}$ portion have been considered.

\section{PSPC Pulse Height Spectra of Active Stars}

A major advantage of the ROSAT all-sky survey is the availability of pulse height spectra, which define up to four more or less independent bands over the PSPC band pass. Count statistics limits the useful number of bands often to two, a soft band $(0.1-0.28 \mathrm{keV})$ and a hard band $(0.5-2.0 \mathrm{keV})$. The X-ray sky looks quite different in those two bands. X-ray emission from the quiet $S$ un arises from plasma in active regions with temperatures of $\sim 2-310^{6} \mathrm{~K}$; such plasma emits predominantly in the soft PSPC band with little energy appearing at energies above $1 \mathrm{keV}$. Higher energy emission in the Sun is encountered only during flares, i.e., not in a steady but rather in transient form. Essentially all active stars are detected in ROSAT's hard band; the nearby active stars also exhibit strong emission in the soft band. These emission patterns are indicative of plasma with temperatures of $10^{7} \mathrm{~K}$ or more, and the ROSAT all-sky survey results thus show the ubiquity of such hot 
plasma in active star coronae. It is almost certain that this high temperature emission is radiated in a steady fashion and not in the form of (also existing) flares.

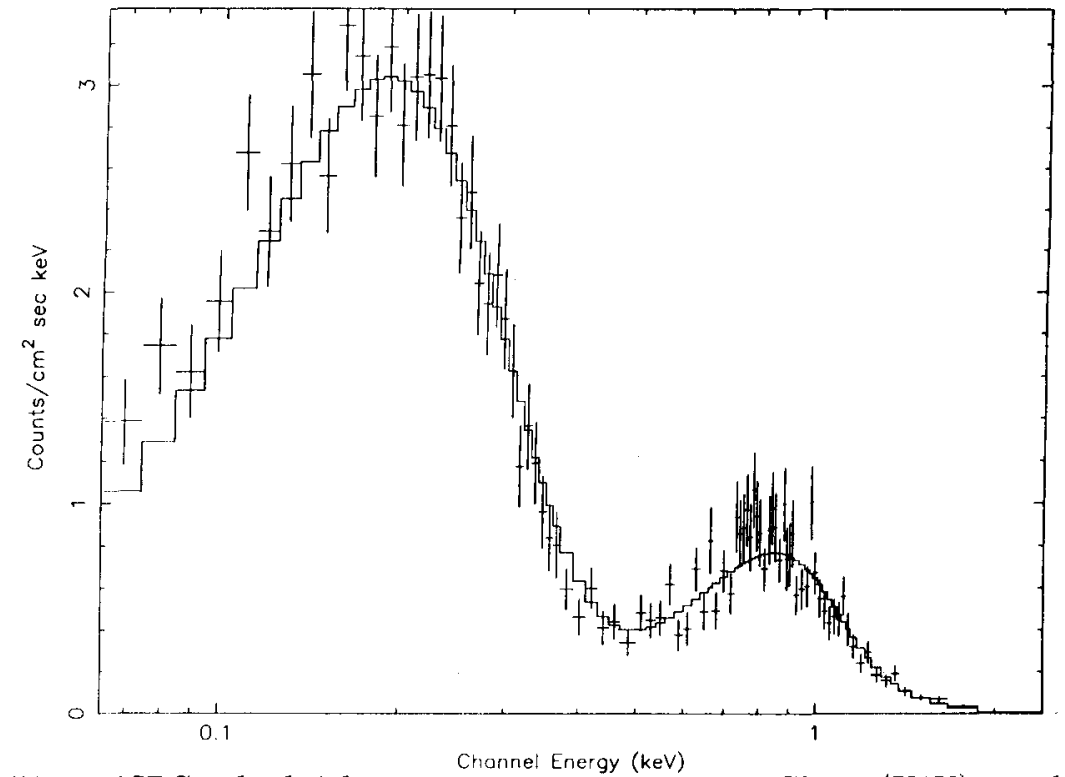

Fig.3: PSPC pulse height spectrum of the active star G1 517 (KOV) together with the best fit two component spectral model.

As an example of a pulse height spectrum of an active star I will present pointed observations of the star Gl 517 (cf., figure 3), which is typical for the apperance of late type active stars in the ROSAT PSPC. When fitting such spectra with thermal line spectra (cf., Schmitt et al. 1990), it turns out to be impossible to obtain acceptable fits with a single isothermal spectral component; such isothermal models correspond to local minima in the fitting process, but given spectra with sufficient count statistics no acceptable fits can be obtained (for G1 517 one finds $\chi_{r e d}^{2}=5.6$ at $\log T=6.43$ ). When two components are included (cf., figure 3), one finds $\chi_{\text {red }}^{2}=1.09$ with $\log T_{\text {low }}=6.32$ and $\log T_{\text {high }}=6.98$, fully confirming the results obtained by Schmitt et al. (1990) in their comprehensive study of X-ray temperatures determined from Einstein Observatory IPC spectra. It should be emphasised that we use such two-component models only to obtain a simple analytical description of the observed pulse height data and we are currently in the process of investigating which constraints can be placed on the differential emission measure distributions of late-type stars in general.

\section{All-sky Survey Lightcurves of Stellar Coronae}

Lastly, the observing mode during the all-sky survey offers interesting possibilities to explore the time variability of coronal X-ray emission. The all-sky survey was carried out in such a way that at any given time a great circle on the sky was scanned. The scan period was linked to the orbital period of $\sim 96$ minutes; any particular region of the sky was viewed for about 25 seconds during a single scan. Any particular source was scanned for at least two days (corresponding about about 30 scans) and up to thirty days and more in the vicinity of the ecliptic poles. The scan great circle was at approximately 90 
degrees from the Sun and moved with the solar motion. In this fashion the whole sky was covered within six months. Therefore the all-sky survey measurements thus represent a light curve covering the time scales of long duration flares (i.e., a few hours) as well as that of stellar rotation (i.e., half a day to a month). For sources at favorable locations near the ecliptic poles such as AB Dor, Gl 687, $\omega$ Dra the ROSAT all-sky survey produced a hitherto unprecedented time series spanning many rotational periods of these objects. Interestingly, so far no convincing examples for rotational modulation could be found in the all-sky survey. Flares, on the other hand, were observed in abundance, for example, on nearby flare stars such as Prox Cen, on RS CVn systems such as HR 1099 and even on rather distant flare stars in open clusters. As an example I show the light curve of the RS CVn system HR 1099 (cf., fig. 4); quite surprisingly, the system stayed relatively constant during the all-sky survey observations, only showing one massive flare almost at the beginning of the observations.

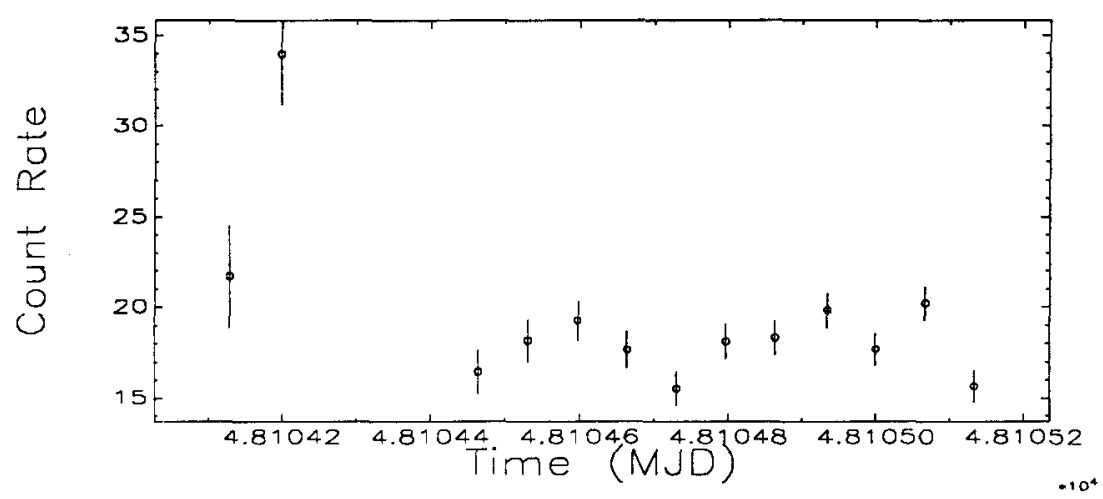

Fig.4: ROSAT all-sky survey light curve of the active RS CVn system HR 1099. Note the massive short duration flare at the beginning of the survey coverage.

\section{References}

Caillault, J.-P. and Helfand, D.., 1989, Ap. J. Letters, 338, L57.

Fleming, T.A., 1991, personal communication.

Gliese, W., 1969, Catalogue of Nearby Stars, Veröff. d. Astron. Recheninst., Heidelberg, 22.

Hoffleit, D. and Jaschek, C., 1982, The Bright Star Catalogue, (New Haven: Yale Univ. Obs.).

Micela, G., Sciortino, S., Vaiana, G.S., Harnden, F.R., Jr., Rosner, R., and Schmitt, J.H.M.M. 1990, Ap. J., 290, 307.

Schmitt, J.H.M.M., Collura, A., Sciortino, S., Vaiana, G.S., Harnden, F.R.Jr., and Rosner, R., 1990, Ap. J., 365, 704 . 